TITLE:

\title{
BEHAVIOR OF RADIALLY SYMMETRIC SOLUTIONS OF A SYSTEM RELATED TO CHEMOTAXIS
}

AUTHOR(S):

NAGAI, TOSHITAKA; SENBA, TAKASI

CITATION:

NAGAI, TOSHITAKA ...[et al]. BEHAVIOR OF RADIALLY SYMMETRIC SOLUTIONS OF A SYSTEM RELATED TO CHEMOTAXIS. 数理解析研究所講究録 1996, 973: 32-39

ISSUE DATE:

1996-11

URL:

http://hdl.handle.net/2433/60746

RIGHT: 


\title{
BEHAVIOR OF RADIALLY SYMMETRIC SOLUTIONS OF A SYSTEM RELATED TO CHEMOTAXIS
}

\author{
TOSHITAKA NAGAI永井 敏隆 \\ Department of Mathematics, Kyushu Institute of Technology, Tobata, Kitakyushu 804 \\ JAPAN \\ TAKASI SENBA 仙葉 隆 \\ Department of Applied Mathematics, Miyazaki University, Kibana, Miyazaki 889-21 \\ JAPAN
}

\section{Introduction}

We consider time-global existence and blow-up of solutions of the following system related to chemotaxis

$$
\begin{cases}b_{t}=\nabla \cdot(\nabla b-\chi b \nabla \phi(s)) & \text { in } \Omega \times(0, \infty), \\ 0=\Delta s-s+b & \text { in } \Omega \times(0, \infty),\end{cases}
$$

under the conditions

$$
\begin{cases}\frac{\partial b}{\partial n}=\frac{\partial s}{\partial n}=0 & \text { on } \quad \partial \Omega \times(0, \infty) \\ b(\cdot, 0)=b_{0} & \text { in } \Omega\end{cases}
$$

where $\chi$ is a postive constant and $\phi$ is a smooth function on $(0, \infty)$ with $\phi^{\prime}>0$. The system is a simplified Keller-Segel model. Keller-Segel model was introduced by Keller and Segel [11] to describe the initiation of chemotactic aggregation of cellular slime molds. On Keller-Segel model and simplified Keller-Segel models, time-local existence of the solutions has been studied by [19] and blow-up of the solutions has been studied by $[4,10,9,14,18]$.

The domain $\Omega$ and the non-trivial initial function $b_{0}$ are only confined to the following case:

(A1) $\Omega$ is the open ball of radius $L$ with center at the origin in $\mathbf{R}^{N}$.

(A2) $b_{0}$ is smooth and nonnegative on $\bar{\Omega}$, and is radially symmetric when $N \geq 2$.

Under these assumptions, there exists a unique solution $(b(x, t), s(x, t))$ to $(1.1)$ and (1.2) defined maximal interval of existence $\left[0, T_{\max }\right)$, which is radially symmetric in $x$ when $N \geq 2$, smooth in $\bar{\Omega} \times\left(0, T_{\max }\right)$ and $b(x, t)>0, s(x, t)>0$ for $(x, t) \in \Omega \times\left(0, T_{\max }\right)$ . If $T_{\max }<\infty$,

$$
\limsup _{t \rightarrow T_{\max }}\left(\|b(\cdot, t)\|_{L^{\infty}}+\|s(\cdot, t)\|_{L^{\infty}}\right)=\infty
$$


by which we mean that $(b(x, t), s(x, t))$ blows up in finite time.

Theorem 1 Let $N=1$ and $\phi$ be smooth on $(0, \infty)$. Then the solution $(b, s)$ to $(1.1)$, (1.2) is globally bounded, that is, $T_{\max }=\infty$ and $(b, s)$ satisfies

$$
\sup _{t \geq 0}\left(\|b(\cdot, t)\|_{L^{\infty}}+\|s(\cdot, t)\|_{L^{\infty}}\right)<\infty
$$

We put

$$
M_{a}(t)=\int_{\Omega} b(x, t)|x|^{a} d x \quad \text { for } 0 \leq t<T_{\max },
$$

where $a$ is a positive constant. That is called the moment of order $a$, of $b(\cdot, t)$.

Theorem 2 Assume $\phi(s)=s^{p}(p>0)$, (A1) and (A2).

(1) $N=2$ :

(a) If $0<p<1$, then the solution is globally bounded in time.

(b) $p=1$ :

(i) If $\left\|b_{0}\right\|_{L^{1}}<8 \pi / \chi$, then the solution is globally bounded in time.

(ii) If $\left\|b_{0}\right\|_{L^{1}}>8 \pi / \chi$ and $M_{2}(0)$ is sufficiently small, then the solution blows up in finite time.

(c) If $p>1$ and $M_{2}(0)$ is sufficiently small, then the solution blows up in finite time.

(2) If $N \geq 3$ and $M_{(N-2) p+2}(0)$ is sufficiently small, then the solution blows up in finite time.

Theorem 3 Assume $\phi(s)=\log s$, (A1) and (A2).

(1) If $N=2$, then the solution is globally bounded in time.

(2) $N \geq 3$ :

(a) If $\chi<2 /(N-2)$, then the solution is globally bounded in time.

(b) If $\chi>2 N /(N-2)$ and $M_{2}(0)$ is sufficiently small, then the solution blows up in finite time.

\section{Time-global existence and boundedness}

The purpose in this section is to sketch the proofs of Theorem 1 and (i) in Theorems 2 and 3.

Let $G$ be the Green function of $-\Delta+1$ in $\Omega$ with homogeneous Neumann boundary conditions. For $N \geq 2$ we put

$$
E(r)=(2 \pi)^{-N / 2} r^{(2-N) / 2} \kappa_{(N-2) / 2}(r) \quad \text { for } r>0,
$$

where $\kappa_{\nu}$ is the modified Bessel function of the second kind of order $\nu$ (see [13]). $E$ is a fundamental solution of $-\Delta+1$.

For the solution $(b, s)$ to $(1.1),(1.2)$ define the functions $S$ and $B$ by 


$$
S(r, t)=\int_{|x| \leq r} s(x, t) d x, \quad B(r, t)=\int_{|x| \leq r} b(x, t) d x
$$

for $0 \leq r \leq L$ and $0 \leq t<T_{\max }$, respectively. $B$ and $S$ satisfy

$$
\begin{aligned}
\frac{\partial B}{\partial t} & =r^{N-1} \frac{\partial}{\partial r}\left(r^{1-N} \frac{\partial B}{\partial r}\right)+\frac{\chi}{\omega_{N}}(B-S) \phi^{\prime}(s) r^{1-N} \frac{\partial B}{\partial r} \\
0 & =r^{N-1} \frac{\partial}{\partial r}\left(r^{1-N} \frac{\partial S}{\partial r}\right)-S+B
\end{aligned}
$$

for $0<r<L$ and $0<t<T_{\max }$, and

$$
B(0, t)=S(0, t)=0, \quad B(L, t)=S(L, t)=\left\|b_{0}\right\|_{L^{1}}
$$

where $\omega_{N}$ is the surface area of the unit sphere $S^{N-1}$ in $\mathbf{R}^{N}$.

In order to show the boundedness and time-global existence of solutions $(b, s)$ to $(1.1)$, (1.2), we begin with the following lemmas. These lemmas are shown by the arguments similar to those in [14] and [18], respectively, so we omit the proofs. In what follows, $C$ denotes a generic positive constant depending on $L$ and $N$.

Lemma 2. 1 Let $N \geq 2$. Then

$$
s(x, t) \geq C\left\|b_{0}\right\|_{L^{1}} \quad \text { for } x \in \bar{\Omega} \text { and } t \in\left(0, T_{\text {max }}\right) .
$$

Lemma 2. 2 . If the following condition

$$
\sup _{0 \leq t<T_{\max }}\|s(\cdot, t)\|_{L^{\infty}}<\infty, \quad \sup _{0 \leq t<T_{\max }}\|\nabla \phi(s(\cdot, t))\|_{L^{\infty}}<\infty
$$

holds, then $T_{\max }=\infty$ and

$$
\sup _{t>0}\|b(\cdot, t)\|_{L^{\infty}}<\infty .
$$

For the following lemma, see [18].

Lemma 2. 3 Let $N \geq 2$. Then the following holds :

$$
B(|x|, t) E(|x|) \leq s(x, t) \leq C\left\|b_{0}\right\|_{L^{1}} E(|x|) \quad \text { in } \Omega \backslash\{0\} \times\left(0, T_{\max }\right) .
$$

Sketch of proofs of Theorem 1 and (i) in Theorems 2 and 3. By Lemmas 2. 1 , 2.2 and Appendixes in [14] and [18], it suffices to show that

$$
\sup _{0 \leq t<T_{\max }}\|s(\cdot, t)\|_{L^{\infty}}<\infty, \quad \sup _{0 \leq t<T_{\max }}\|\nabla s(\cdot, t)\|_{L^{\infty}}<\infty .
$$

In the case of $N=1,(2.4)$ is shown by the arguments similar to those in [14]. Hence we will prove (2.4) in the case of $N \geq 2$.

We put

$$
\Phi(u)= \begin{cases}p\left\|b_{0}\right\|_{L^{1}}^{p} u^{p-1} & \text { in the case of Theorem } 2, \\ u^{-1} & \text { in the case of Theorem } 3\end{cases}
$$


for $u>0$. It follows from Lemma 2.3, (2.2) and $\partial B / \partial r \geq 0$ that $B$ satisfies

$$
\frac{\partial B}{\partial t} \leq r^{N-1} \frac{\partial}{\partial r}\left(r^{1-N} \frac{\partial B}{\partial r}\right)+\frac{\chi}{\omega_{N}} \Phi(E) r^{1-N} \frac{\partial B}{\partial r} .
$$

We can construct the function $W(r)$ such that

$$
\begin{aligned}
& \quad W(r) \sim r^{N} \text { as } r \rightarrow 0, \\
& \left\|b_{0}\right\|_{L^{1}}<W(L) \quad \text { and } \quad B(r, 0) \leq W(r) \quad \text { for } 0 \leq r \leq L,
\end{aligned}
$$

and that

$$
0 \geq r^{N-1} \frac{d}{d r}\left(r^{1-N} \frac{d W}{d r}\right)+\frac{\chi}{\omega_{N}} B \phi^{\prime}(s) r^{1-N} \frac{d W}{d r} \text { for } 0<r<L .
$$

Hence, the comparison theorem yields that

$$
B(r, t) \leq W(r) \quad \text { for } 0 \leq r \leq L, 0 \leq t<T_{\max }
$$

which implies $B(r, t) \leq C r^{N}$.

Since $B(r, t) \leq C r^{N}$ for $0 \leq r \leq L$ and $0 \leq t<T_{\text {max }}$, it follows from (2.3) that

$$
S(r, t) \leq C r^{N} \quad \text { for } 0 \leq r \leq L, 0 \leq t<T_{\text {max }} .
$$

Then we have that

$$
|\nabla s(x, t)|=\frac{|S(|x|, t)-B(|x|, t)|}{\omega_{N}|x|^{N-1}} \leq C
$$

for $x \in \Omega$ and $0 \leq t<T_{\max }$. The boundedness of $\|s(\cdot, t)\|_{L^{\infty}}$ with respect to $t \in\left[0, T_{\max }\right)$ follows from the estimate above of $|\nabla s|$ and

$$
\min _{x \in \bar{\Omega}} s(x, t) \leq \frac{\left\|b_{0}\right\|_{L^{1}}}{|\Omega|} \quad \text { for } 0 \leq t<T_{\max }
$$

where $|\Omega|$ is the volume of $\Omega$. Thus the proofs of (i) of Theorems 2 and 3 are complete.

\section{Blow-up of solutions}

The purpose in this section is to show the blow-up of solutions for the system (1.1), (1.2) in the case of $N \geq 2$.

In order to show the blow-up of solutions $(b, s)$ to $(1.1),(1.2)$ in [14] and [18], a differential inequality on a moment $M_{k}(t)$ of $b$ is constructed by use of some estimates of $s$, and under some conditions on $b_{0}$ it is shown that the moment of $b$ converges to 0 as $t$ tends some $T_{0} \in(0, \infty)$ by use of the differential inequality.

The following lemma is an immediate consequence of Hölder's inequality.

Lemma 3. 1 Let $f$ be an integrable function on $\Omega$, and $p_{1}, p_{2}$ and $p_{3}$ be numbers satisfying $0 \leq p_{1}<p_{2}<p_{3}$. Then

$$
\int_{\Omega}|f||x|^{p_{2}} d x \leq\left\{\int_{\Omega}|f||x|^{p_{1}} d x\right\}^{\left(p_{3}-p_{2}\right) /\left(p_{3}-p_{1}\right)}\left\{\int_{\Omega}|f||x|^{p_{3}} d x\right\}^{\left(p_{2}-p_{1}\right) /\left(p_{3}-p_{1}\right)} .
$$


Let $S$ and $B$ be the same functions as in (2.1). The following lemmas are stated in [15] and [18].

Lemma 3. 2 The inequality holds:

$$
\begin{aligned}
\frac{d}{d t} M_{k}(t) \leq & k(k+N-2) \int_{\Omega} b(x, t)|x|^{k-2} d x \\
& +\frac{k \chi}{\omega_{N}} \int_{\Omega} \phi^{\prime}(s(x, t)) b(x, t)\{S(|x|, t)-B(|x|, t)\}|x|^{k-N} d x
\end{aligned}
$$

on $\left(0, T_{\max }\right)$, where $k \geq 2$.

Lemma 3.3 Let $N \geq 3$. There exists a positive constant $\delta$ such that

$$
\frac{\partial}{\partial r}\left(r^{N-1} s(x, t)\right) \geq 0 \quad \text { in }\left\{x \in R^{N}:|x| \leq \delta\right\} \times\left(0, T_{\text {max }}\right),
$$

where $r=|x|$.

Lemma 3. 4 Let $N \geq 2$. Then the following holds:

$$
s(x, t) \leq \frac{1}{\omega_{N}|x|^{N-1}} \int_{|y|=|x|} E(|x-y|) d \sigma\left\|b_{0}\right\|_{L^{1}}+\int_{\Omega} K(x, y) b(y, t) d y
$$

in $\Omega \backslash\{0\} \times\left(0, T_{\max }\right)$.

Sketch of proof of (ii) of Theorem 2. Let $k=(N-2) p+2$. In order to prove the theorem, it suffices to show the following inequality

$$
\begin{aligned}
\frac{d}{d t} M_{k}(t) \leq & k(k+N-2)\left\|b_{0}\right\|_{L^{1}}^{2 / k} M_{k}(t)^{(k-2) / k} \\
& +C\left\|b_{0}\right\|_{L^{1}}^{p+(k-2) / k} M_{k}(t)^{2 / k}-C\left\|b_{0}\right\|_{L^{1}}^{p+1}
\end{aligned}
$$

for $t \in\left(0, T_{\max }\right)$. In fact, if $M_{k}(0)$ is sufficiently small so that the right-hand side of (3.1) is negative at $t=0$, there exists $T_{0} \in(0, \infty)$ such that

$$
M_{k}(t) \rightarrow 0 \text { as } t \rightarrow T_{0}
$$

Hence, $T_{m a x}$ must be finite and $T_{m a x} \leq T_{0}$. By Appendixes in [14] and [18], we have

$$
\limsup _{t \rightarrow T_{\max }}\|b(\cdot, t)\|_{L^{\infty}}=\infty
$$

Let us first show (3.1) in the case of $p \geq 1$. Using Lemmas 2.3 and the properties of the fundamental solution, we obtain that

$$
\int_{\Omega} s^{p-1}(x, t) b(x, t) B(|x|, t)|x|^{k-N} d x \geq C\left\|b_{0}\right\|_{L^{1}}^{p+1} .
$$

and that

$$
S(|x|, t) \leq C\left\|b_{0}\right\|_{L^{1}}|x|^{2}
$$


It follows from Lemma 2. 3 and (3.3) and the properties of the fundamental solution that

$$
\int_{\Omega} s^{p-1}(x, t) b(x, t) S(|x|, t)|x|^{k-N} d x \leq C\left\|b_{0}\right\|_{L^{1}}^{p} M_{2}(t)
$$

Lemma 3. 2 together with (3.2), (3.4) and Lemma 3. 1 yields (3.1).

Let us consider the case $0<p<1$. By Lemmas 2.3 and the properties of the fundamental solution, we have

$$
\begin{aligned}
& \int_{\Omega} s^{p-1}(x, t) b(x, t) B(|x|, t)|x|^{k-N} d x \\
& \geq C\left\|b_{0}\right\|_{L^{1}}^{p-1} \int_{\Omega} b(x, t) B(|x|, t) d x=\frac{C}{2}\left\|b_{0}\right\|_{L^{1}}^{p+1} .
\end{aligned}
$$

It follows from Lemmas 2.3 and 3.3 and the properties of the fundamental solution that

$$
\int_{|x| \leq \delta} s^{p-1}(x, t) b(x, t) S(|x|, t)|x|^{k-N} d x \leq C\left\|b_{0}\right\|_{L^{1}}^{p} \int_{|x| \leq \delta} b(x, t)|x|^{2} d x .
$$

By Lemma 2. 1 and (3.3), we have

$$
\begin{aligned}
& \int_{\delta \leq|x| \leq L} s^{p-1}(x, t) b(x, t) S(|x|, t)|x|^{k-N} d x \\
& \leq C\left\|b_{0}\right\|_{L^{1}}^{p} \int_{\delta \leq|x| \leq L} b(x, t)|x|^{k-N+2} d x \\
& \leq C\left\|b_{0}\right\|_{L^{1}}^{p} \delta^{k-N} \int_{\delta \leq|x| \leq L} b(x, t)|x|^{2} d x .
\end{aligned}
$$

Combining (3.6) with (3.7) yields that

$$
\int_{\Omega} s^{p-1}(x, t) b(x, t) S(|x|, t)|x|^{k-N} d x \leq C\left\|b_{0}\right\|_{L^{1}}^{p} M_{2}(t) .
$$

By (3.5) and (3.8), the similar argument to that in the case of $p \geq 1$ gives us (3.1). Thus the proof is complete.

Sketch of proof of (ii) in Theorem 3. Observe that it follows from Lemma 3.4 and the properties of the fundermental solution that for $0 \leq t<T_{\max }$ and $0<|x| \leq L / 2$,

$$
s(x, t) \leq\left\{\frac{1}{\omega_{N}(N-2)|x|^{N-2}}+C\left(|x|^{3-N}+1\right)\right\}\left\|b_{0}\right\|_{L^{1}} .
$$

For $0 \leq t<T_{\max }$ and $0<\delta \leq L / 2$, we then have that

$$
\begin{aligned}
& \int_{\Omega} b(x, t) B(|x|, t) \frac{1}{|x|^{N-2} s(x, t)} d x \\
& \geq \frac{1}{\left\|b_{0}\right\|_{L^{1}}}\left\{\frac{1}{(N-2) \omega_{N}}+C \delta\right\}^{-1} \int_{|x| \leq \delta} b(x, t) B(|x|, t) d x
\end{aligned}
$$




$$
\begin{aligned}
& \geq \frac{(N-2) \omega_{N}}{2(1+C \delta)\left\|b_{0}\right\|_{L^{1}}} B(\delta, t)^{2} \\
& \geq \frac{(N-2) \omega_{N}}{2(1+C \delta)\left\|b_{0}\right\|_{L^{1}}}\left(\left\|b_{0}\right\|_{L^{1}}-\frac{1}{\delta^{2}} M_{2}(t)\right)_{+}^{2} \\
& \geq \frac{(N-2) \omega_{N}}{2(1+C \delta)}\left(\left\|b_{0}\right\|_{L^{1}}-\frac{2}{\delta^{2}} M_{2}(t)\right),
\end{aligned}
$$

where $(\cdot)_{+}=\max \{\cdot, 0\}$. It follows from Lemma 3.3 that

$$
\begin{aligned}
& \int_{\Omega} b(x, t) S(|x|, t) \frac{1}{|x|^{N-2} s(x, t)} d x \\
& \quad=\omega_{N} \int_{|x| \leq \delta} b(x, t)|x|^{2} d x+\int_{\delta \leq|x| \leq L} b(x, t) S(|x|, t) \frac{1}{|x|^{N-2} s(x, t)} d x \\
& \quad \leq C M_{2}(t)
\end{aligned}
$$

in $\left(0, T_{\max }\right)$. Hence, combining Lemma 3.2 with (3.9) and (3.10) concludes that

$$
\frac{d}{d t} M_{2}(t) \leq\left\{2 N-\frac{(N-2) \chi}{1+C \delta}\right\}\left\|b_{0}\right\|_{L^{1}}+C \chi\left(1+\delta^{-2}\right) M_{2}(t)
$$

on $\left(0, T_{\max }\right)$. Suppose that $\delta$ is sufficiently small so that $2 N(1+C \delta)-(N-2) \chi<0$. Using the argument similar to that in the sketch of proof of Theorem 2 , then we have the proof.

\section{References}

[1] P. Biler and T. Nadzieja, Existence and nonexistence of solutions for a model of gravitational interactions of particles, I, Colloq. Math., 66 (1994), 319-334.

[2] P. Biler, Existence and nonexistence of solutions for a model of gravitational interactions of particles, III, Colloq. Math., 68 (1995), 229-239.

[3] S. Childress, Chemotactic collapse in two dimensions, Lecture Notes in Biomath., vol. 55, Springer, Berlin-Heidelberg-New York, 1984, 61-66.

[4] S. Childress and J. K. Percus, Nonlinear aspects of chemotaxis, Math. Biosci., 56 (1981), 217-237.

[5] J. I. Diaz and T. Nagai, Symmetrization in a parabolic-elliptic system related to chemotaxis, Adv. Math. Sci. Appl., 5 (1995), 659-680.

[6] G. B. Folland, Introduction to Partial Differential Equations, Princeton University Press, Princeton, New Jersey, 1976.

[7] P. R. Garabedian, Partial Differential Equations, John Wiley \& Sons, New York, 1964.

[8] D. Henry, Geometric Theory of Semilinear Parabolic Equations, Lecture Notes in Math., vol. 840, Springer, Berlin-Heidelberg-New York, 1981. 
[9] M. A. Herrero and J. J. L. Velázquez, Singularity patterns in a chemotaxis model, Math. Ann., to appear.

[10] W. Jäger and S. Luckhaus, On explosions of solutions to a system of partial differential equations modelling chemotaxis, Trans. Amer. Math. Soc., 329 (1992), 819-824.

[11] E. F. Keller and L. A. Segel, Initiation of slime mold aggregation viewed as an instability, J. Theor. Biol., 26 (1970), 399-415.

[12] I. R. Lapidus and M. Levandowsky, Modeling chemosensory responses of swimming eukarvotes, Biological Growth and Spread, Proceedings, Heidelberg 1979, Lecture Notes in Biomathematics, vol. 38, Springer-Verlag, 1980.

[13] T. Myint-U and L. Debnath, Partial Differential Equations for Scientists and Engineers, North-Holland, New York, 1987.

[14] T. Nagai, Blow-up of radially symmetric solutions to a chemotaxis system, Adv. Math. Sci. Appl., 5 (1995), 581-601.

[15] T. Nagai and T. Senba, Global existence and blow-up of radially symmetric solutions to a parabolic-elliptic system to chemotaxis, preprint.

[16] V. Nanjundiah, Chemotaxis, signal relaying, and aggregation morphology, J. Theor. Biol., 42 (1973), 63-105.

[17] R. Schaaf, Stationary solutions of chemotaxis systems, Trans. Amer. Math. Soc., 292 (1985), 531-556.

[18] T. Senba, Blow-up of radially symmetric solutions to some systems of partial differential equations modelling chemotaxis, Adv. Math. Sci. Appl., to appear.

[19] A. Yagi, Norm behavior of solutions to the parabolic system of chemotaxis, preprint. 